\section{Therapy-related myeloid neoplasms as a concerning complication in acute promyelocytic leukemia}

\author{
María del Carmen Vicente-Ayuso, ${ }^{1,2}$ \\ María García-Roa, ${ }^{1,2}$ \\ Ataúlfo González-Fernández, ${ }^{1,2}$ \\ Ana María Álvarez-Carmona, ${ }^{1}$ \\ Celina Benavente-Cuesta, ${ }^{1}$ \\ Marta Mateo-Morales, ${ }^{1}$ \\ Cristina Pérez-López, ${ }^{1}$ \\ Ascensión Peña-Cortijo, ${ }^{1}$ \\ Marta Polo Zarzuela, ${ }^{1}$ \\ Laura Gutiérrez, ${ }^{2}$ \\ Rafael Martínez-Martínez ${ }^{1}$ \\ ${ }^{1}$ Servicio de Hematología y \\ Hemoterapia, Hospital Clínico San \\ Carlos; ${ }^{2}$ Department of Hematology, \\ Instituto de Investigación Sanitaria San \\ Carlos (IdISSC), Hospital Clínico San \\ Carlos, Madrid, Spain
}

\begin{abstract}
Acute promyelocytic leukemia (APL) has become a highly curable malignant disease after the introduction of all transretinoic acid (ATRA) to chemotherapy treatment. However, the risk to develop therapy-related myeloid neoplasms (t-MN) has become a matter of concern, as APL patients are otherwise expected to have a good prognosis. We report a patient with APL who achieved complete remission after chemotherapy induction with anthracycline and ATRA, followed by consolidation and maintenance chemotherapy. Two years later, the patient developed t-AML, with $M L L$ rearrangements, without any evidence of relapse of the APL original clone. The increasing incidence of $\mathrm{t}-\mathrm{MN}$ in oncohematological patients is partly due to the development of safer, more efficient or targeted therapies, which allow better outcomes and lengthened survival amongst treated patients. The identification of genetic factors, mechanisms or prognostic biomarkers in t-MN might open new windows for the development of personalized targeted therapy regimes in this underserved patient population.
\end{abstract}

\section{Introduction}

Acute promyelocytic leukemia (APL), also known as acute myeloid leukemia (AML) M3 subtype of the FrenchAmerican-British (FAB) classification, is a highly fatal subtype of AML. It is caused by $t(15 ; 17)$, a translocation that results in the fusion of the promyelocytic leukemia $(P M L)$ gene on chromosome 15 to the retinoic acid receptor alpha $(R A R A)$ gene on chromosome 17, which also serves as a clone specific molecular marker for diagnosis and monitoring. ${ }^{1}$

This unique molecular abnormality allows targeted therapy, making APL highly curable. In the past, anthracycline and cytosine arabinoside (Ara-C) based combination chemotherapy yielded cure rates of only $35-$ $40 \%$ in APL patients, whereas the introduction in the early 1990s of all trans-retinoic acid (ATRA) to the chemotherapy regime, in combination with anthracycline-Ara $\mathrm{C}$ or anthracycline alone, has improved the cure rate to $80-90 \%$. However, recent reports suggest an increasing risk in APL patients to develop therapy-related myeloid neoplasms $(\mathrm{t}-\mathrm{MN}){ }^{2-7} \mathrm{t}-\mathrm{MN}$ are clonal hematopoietic disorders that arise due to iatrogenic somatic mutations that increase proliferative capacity and survival advantage of the affected hematopoietic progenitors. They are considered as a subgroup of its own in the revised 2016 WHO classification, comprising myelodysplastic syndrome (t-MDS) and acute myeloid leukemia (t-AML) patients who were exposed to cytotoxic or radiation therapy for an unrelated malignancy or an autoimmune disease (i.e. multiple sclerosis or rheumatologic disease).$^{8}$ Both, t-MDS and t-AML are combined in one entity due to their similar pathogenesis, rapid progression from t-MDS to t-AML, and their equally poor prognosis with no differences in outcome. Moreover, t-MN should be distinguished from AML with myelodysplasia (secondary AML), which is diagnosed either when $50 \%$ or more of the bone marrow cells are dysplastic in at least two lineages, when the patient had a previous diagnosis of MDS or MDS/MN or when myelodysplasia-associated cytogenetic aberrations are present. ${ }^{3,4}$

Hereby, we report a patient with APL who presented complete clinical and molecular remission after chemotherapy. Two years after diagnosis, the patient developed t-AML with $M L L$ rearrangements, without any evidence of relapse of the APL original clone. This clinical manifestation, i.e. $\mathrm{t}-$ $\mathrm{MN}$, though rare, has been increasingly diagnosed and is a matter of concern in treated APL patients, who are otherwise expected to have a good prognosis after targeted therapy.
Correspondence: María del Carmen VicenteAyuso, Hospital Clínico San Carlos, Servicio de Hematología y Hemoterapia, Planta Primera Sur, Marco C/Profesor Martín Lagos S/N, 28040 Madrid, Spain.

Tel.: +34.91.330.3321 - Fax: +34.91.330.3322 E-mail: mentxu888@gmail.com

Key words: Acute promyelocytic leukemia, therapy-related myeloid neoplasms, therapyrelated acute myeloid leukemia.

Contributions: MdCVA and MGR contributed equally as the first authors. MdCVA and MGR followed the case, analyzed data and wrote the manuscript. $\mathrm{CBC}$ and $\mathrm{AGF}$ managed the patient, followed the case, analyzed data and revised the manuscript. LGG analyzed data and wrote the manuscript. AMAC, MMM, CPL, APC, MPZ and RMM managed the patient.

Conflict of interest: the authors declare no potential conflict of interest.

Funding: LG was supported by a Ramón y Cajal Fellowship (RYC-2013-12587; Ministerio de Economía y Competitividad, Spain).

Received for publication: 28 April 2017.

Revision received: 21 June 2017

Accepted for publication: 22 June 2017.

This work is licensed under a Creative Commons Attribution-NonCommercial 4.0 International License (CC BY-NC 4.0).

CCopyright M. del Carmen Vicente-Ayuso et al., 2017 Licensee PAGEPress, Italy

Hematology Reports 2017; 9:7204

doi:10.4081/hr.2017.7204

\section{Case Report}

A 46-year-old woman presented with spontaneous bruises and pancytopenia in 2008. Complete Blood Counts (CBC) measured showed a hemoglobin concentration of $10.7 \mathrm{~g} / \mathrm{dL}$, a leukocyte count of $1.2 \times 10^{6} / \mathrm{mL}$ (with differential of $30 \%$ neutrophils, $40 \%$ lymphocytes and $30 \%$ blasts), and a platelet count of $18 \times 10^{6} / \mathrm{mL}$ (Figure $1)$. Screen coagulation was normal. Peripheral blood smear and bone marrow aspiration were suggestive of an infiltration of $90 \%$ of abnormal promyelocytes and blasts (Figure 2A). Bone marrow flow cytometry analysis confirmed the aberrant population $(77.5 \%$ of viable cells $)$ immunophenotyped as $\mathrm{CD} 33^{+} \mid \mathrm{CD} 45^{+}$ $\mid \mathrm{CD} 117^{\text {mid }}$ with a $\mathrm{CD}^{+} 3^{+}$homogeneous scattered pattern and $\mathrm{MPO}^{+}$, and negative for HLA-DR, CD34, CD123, CD14, CD15, 
CD64, CD42A, and GPA (Figure 2B and data not shown). Cytogenetic analyses revealed $\mathrm{t}(15 ; 17)$ and $P M L / R A R A$ rearrangement, without identifying other secondary genetic lesions. She completed chemotherapy treatment according to the Spanish Program of Treatments in Hematology (PETHEMA) 2005 protocol for patients with intermediate risk APL (induction with ATRA and Idarubicin, three cycles of consolidation and maintenance with Mercaptopurine, methotrexate and ATRA), after which complete hematological and molecular remission (CR) was observed (Figure 1).

In September 2010, the patient presented with leucopenia and thrombocytopenia (Figure 1). Cytology and flow cytometry of bone marrow aspirate showed $7 \%$ of blasts with dysplasia AREB-II versus AML that were immunophenotyped as $\mathrm{CD} 117^{+}\left|\mathrm{CD} 45^{+}\right| \mathrm{CD} 33^{++}$and negative for CD34 and HLA-DR, suggestive of an early stage of relapse (Figure 3A). The main differential diagnosis was established between a relapse of APL or a different subtype of AML secondary to treatment (t-AML). APL was discarded since $t(15 ; 17)$ was not detected. A t-AML relapse was confirmed on peripheral blood smear cytology two weeks later (Figure 3B) and bone marrow aspirate containing $70 \%$ of blasts. From the karyotype, a 46XX cell clone containing rearrangements on the $M L L$ gene was identified, with breakpoints in chromosomes 20q12 and 11q23, corresponding to unfavorable European Leukemia NET (ELN) Score. With a diagnosis of t-AML in a patient with previous history of treated APL, she received two cycles of chemotherapy with Idarubicin and Ara-C $(7+3$ induction regimen), reaching $\mathrm{CR}$ with negative minimal residual disease (MRD), and without $M L L$ gene rearrangements, which she maintained after consolidation with Ara-C at high doses. In March 2011, allogenic hematopoietic cell transplantation (HCT) was performed (Figure 1).

After four years suffering serious symptoms related to chronic skin (grade II) and hepatic (grade III) GvHD, she presented with pancytopenia and a suspicion of relapse of t-AML in April 2015, later confirmed on bone marrow cytologic and immunophenotypic studies. She deceased due to a septic shock of respiratory origin before further treatment could be initiated.

\section{Discussion}

The recent increase in the number of reports of $\mathrm{t}-\mathrm{MN}$ ( $\mathrm{t}-\mathrm{AML})$ in treated APL

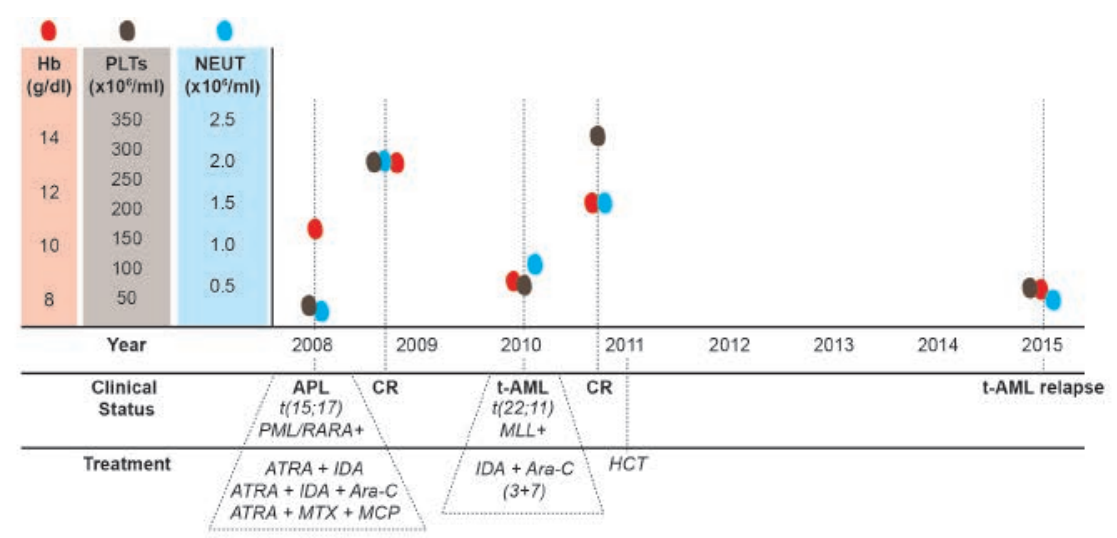

Figure 1. Schematic representation of the evolution of the reported case. The $\mathrm{X}$-axis depicts time (year). The upper part of the scheme shows hemoglobin levels $(\mathrm{Hb}$, red dots and corresponding Y-axis within red-shaded block), platelet counts (PLTs, brown dots and corresponding Y-axis within brown-shaded block) and neutrophil counts (NEUT, blue dots and corresponding Y-axis within blue-shaded block) as measured at the indicated times (vertical dashed lines). In the lower part of the scheme, the clinical status and treatment given at the indicated times (vertical dashed lines) is highlighted. APL: acute promyelocytic leukemia; $t-A M L$ : therapy related acute myeloid leukemia; CR: complete remission; HCT: allogenic hematopoietic cell transplantation.

A

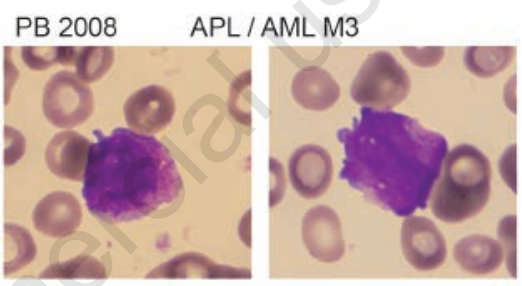

B

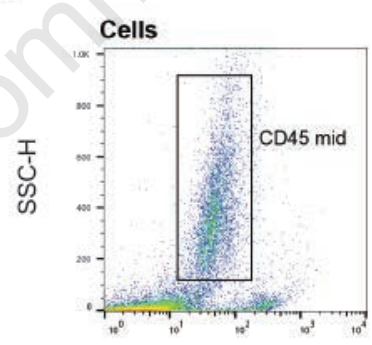

CD45 PerCP

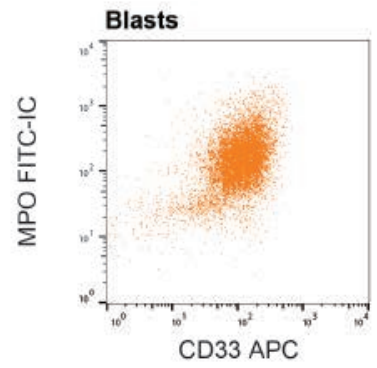

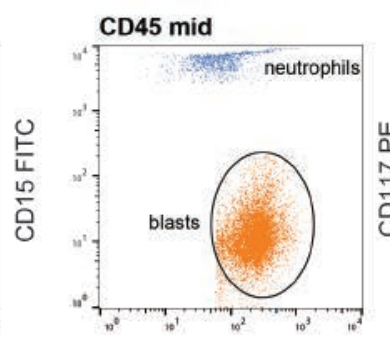

CD33 PE

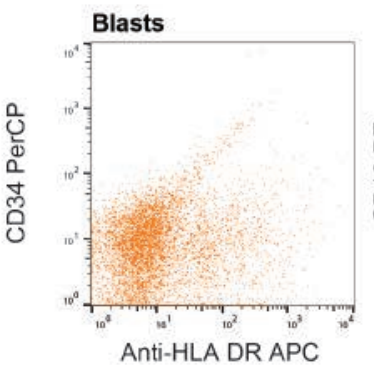

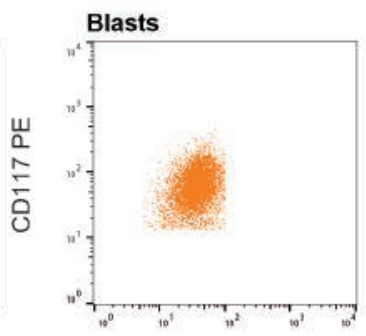

CD45 PerCP

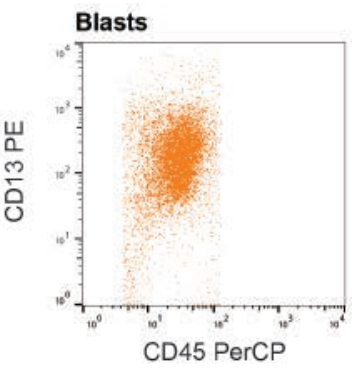

Figure 2. A) Representative pictures of peripheral blood (PB) smear at time of APL (AML M3) diagnosis (Hematoxylin \& Eosin staining, magnification $\times 100)$. B) Flow cytometry analysis of bone marrow aspirate at time of APL diagnosis. Dot plots are depicted: the gated population is indicated on top of each dot plot. The CD45 mid population was plotted against CD15 and CD33, and CD33+CD15- aberrant blasts (orange population in the dot plots) were further analyzed for expression of a series of markers, i.e. surface CD117, CD34, HLA-DR, CD13 and intracellular (IC) MPO. 
patients, allows epidemiologic analysis of its occurrence. Relapse after CR with a different subtype of AML is rare (between 1\% and $6.5 \%$ of reported cases), ${ }^{6}$ while relapse as APL occurs in $10-15 \%$ of the cases. ${ }^{9} \mathrm{t}$ $\mathrm{MN}$ is predominant in women, which can be partly explained by the fact that the most frequent primary malignancy is breast cancer followed by non-Hodgkin lymphoma. The risk to develop t-MN has increased during the last three decades in non-Hodgkin lymphoma, while it has declined in ovarian cancer and multiple myeloma, and it has remained constant in breast cancer and Hodgkin lymphoma. t-MN appears at any age, but the median age at diagnosis is 61 years. ${ }^{2-7}$ It is necessary to consider several covariates responsible for the increased risk of t-MN, including age, time since prior therapy (i.e. latency period), type of chemotherapy and cumulative dose, use of combined modality regimes (chemotherapy and radiation), and dose and volume of radiation applied. In this context, the origin of a t-MN clone, different from the original, could be due to a number of collateral causes. i) The t-MN clone co-exists with the original APL clone at diagnosis, however, not being affected by APL targeted therapy, it overgrows after CR. The incidence of cooccurrence of additional cytogenetic abnormalities (ACA) at initial diagnosis in APL patients has been reported in one-third of analyzed cases. However, there is no evidence that the presence of ACA at the time of diagnosis correlates with the outcome in APL patients. ${ }^{10}$

The high frequency of TP53 mutations found in patients with t-MN (approximately one third of them) supports the notion of cooccurring treatment-resistant clones. It has been reported that malignant clones bearing TP53 mutations require two induction courses to achieve CR, suggesting relative chemotherapy resistance. ${ }^{11}$ Other genes with a mutation frequency of $>5 \%$ in $\mathrm{t}-\mathrm{MN}$ include NPM1, FLT3-ITD, TET2, DNMT3A, STAG2, ABC transporter genes, IDH1, IDH2, RUNX1 and ASXL1 amongst others. $^{2-7,11-14}$

ii) The t-MN clone is induced by therapy, as it is known that the drugs used and the patient genetic susceptibility may play a role. The pathogenesis can be explained by direct induction of an oncogene through chromosomal translocations or genetic instability. Several types of cytotoxic agents, such as topoisomerase II (TOP2) inhibitors, alkylating agents, antimetabolites, and antitubulin agents, in addition to radiotherapy, have been associated with tMN. t-MN that develops after treatment with TOP2 inhibitors, such as etoposide or anthracyclines, are associated to fusion oncogenes such as KTM2A/MLL-MLLT3, have a latency of 2 to 3 years and a better prognosis. In contrast, treatment with alkylating agents has been associated with adverse cytogenetics (i.e. unbalanced aberrations of chromosomes 5 and 7 and/or complex karyotype), a longer latency and a poor prognosis. ${ }^{3-5,7,12-14}$ To date, there is no pathognomonic genetic or morphologic profiling of t-MN. Inherited cancer susceptibility may explain why patients develop more than one type of malignancy, especially under cytotoxic therapy, due to DNA damage in genes such as BRCA1, BRCA2,
BARD1, TP53, RAD51 and HLX1, Fanconi genes, and the anti-apoptotic gene BCL2L10. 2-5,7,12-14

iii) The t-MN clone is induced due to a lineage shift within the myeloid compartment as a virtue of hematopoietic stem cell plasticity. The hematopoietic system homeostasis, affected by the initial disease and/or therapy, responds by inducing potential epigenetic changes favoring abnormal lineage maturation, resulting in non-restrictive proliferation of a non-APL aberrant clone. It has been reported that changes in the microenvironment composition might lead
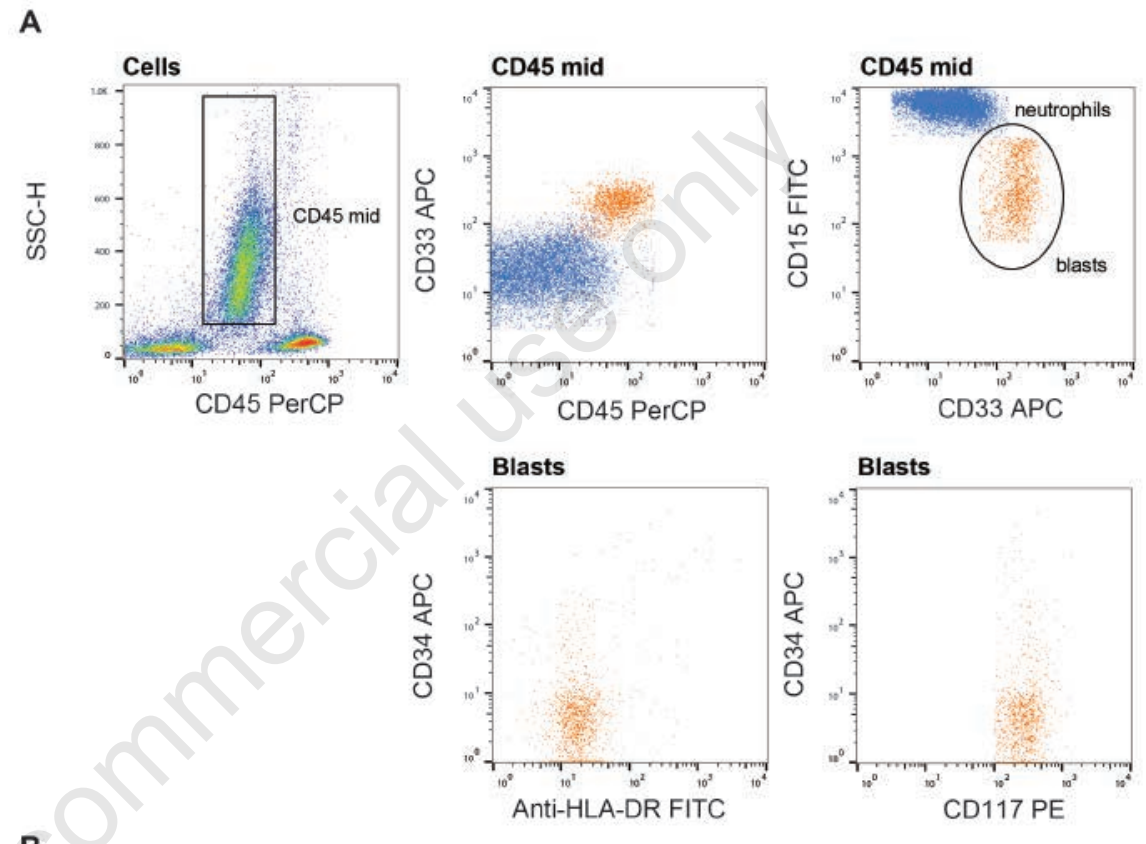

B

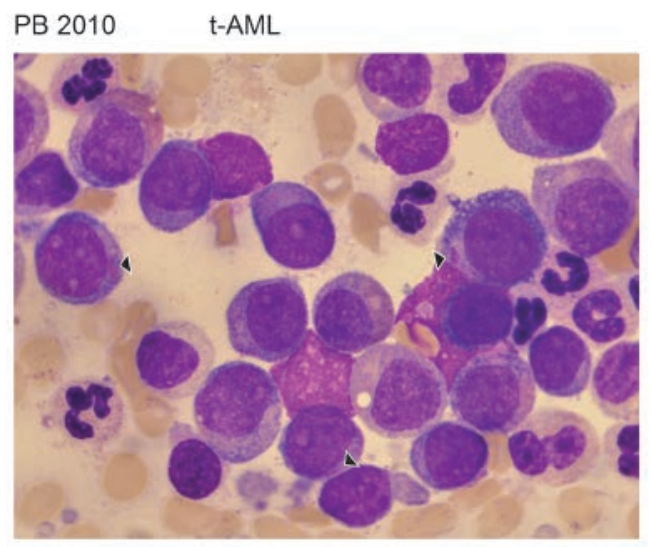

Figure 3. A) Flow cytometry analysis of bone marrow aspirate at time of t-AML diagnosis. Dot plots are depicted: the gated population is indicated on top of each dot plot. The CD45mid population was plotted against $\mathrm{CD} 15$ and $\mathrm{CD} 33$, and $\mathrm{CD} 33^{+} \mathrm{CD} 15^{\mathrm{mid}}$ aberrant blasts (orange population in the dot plots) were further analyzed for expression of a series of markers, i.e. surface CD117, CD34 and HLA-DR. B) t-AML as observed in peripheral blood (PB) smear, showing increased intermediate-size blasts with a basophilic granular cytoplasm, some of them presenting Auer rods (arrowheads), (Hematoxylin \& Eosin staining, magnification $\times 100)$. APL: acute promyelocytic leukemia; $t-A M L$ : therapy related acute myeloid leukemia. 
to disease, although how any of these alterations would promote lineage switching in leukemia is largely unknown. ${ }^{15}$

We report a patient diagnosed with APL (AML M3) who developed t-AML after achieving complete hematological and molecular remission upon targeted therapy. A relapse of the original APL clone was discarded and $M L L$ rearrangements were identified, confirming a different subtype of AML responsible of the patient's t-AML.

The patient was treated with Idarubicin, a topoisomerase II (TOP2) inhibitor, that has been related with t-AML, and $M L L$ rearrangements. The fact that $M L L$ rearrangements were not identified in our patient with the original APL clone at time of diagnosis, together with the timing and cytogenetic aberration of the relapse clone, suggest the patient suffered from t-AML due to TOP2 treatment. At the time our patient was studied, TP53 mutations and other genetic susceptibility associations were not well established, and thus, they were not investigated. A lineage switch hypothesis seems less likely, since genetic aberrations in APL and secondary leukemia are driven by different genetic drivers.

Prognosis of t-MN is poor with a median survival of less than a year. ${ }^{5,7}$ It has been suggested that malignant cells with multiple aberrations are more immunogenic than cells with one or a few aberrations, and thus novel immune-based therapies, such as chimeric antigen-receptor T cells, bi-specific antibodies, and checkpoint inhibitors, should be considered in t-MN with a highrisk genetic profile and their evaluation in clinical trials should be encouraged. ${ }^{4}$ Cytogenetic classification of t-AML should be further evaluated as a potential prognostic marker, and treatment recommendations should be based on performance status and karyotype. Recent unbiased approaches driven by gene expression analysis and Next Generation Sequencing have contributed to the identification of unique biomarkers associated with leukemia, which have the potential to significantly improve the diagnostic and prognostic criteria, and contribute to the continuous adaptation of treatment protocols. ${ }^{13}$

\section{Conclusions}

We can conclude the following: i) $\mathrm{t}$ $\mathrm{MN}$, though rare, is increasing its occurrence as the survival in onco-hematological patients lengthens. It is therefore a matter of concern in treated APL patients, who are otherwise expected to have good prognosis. ii) t-MN prognosis is uniformly dismal. iii) A better understanding of potential genetic factors or mechanisms around the occurrence of t-MN might open new windows for the development of personalized therapy regimes in this underserved patient population.

\section{References}

1. Tallman MS, Altman JK. How I treat acute promyelocytic leukemia. Blood 2009;114:5126-35.

2. Bueso-Ramos CE, Kanagal-Shamanna R, Routbort MJ, Hanson CA. Therapyrelated myeloid neoplasms. Am J Clin Pathol 2015;144:207-18.

3. Ganser A, Heuser M. Therapy-related myeloid neoplasms. Curr Opin Hematol 2017;24:152-8.

4. Heuser M. Therapy-related myeloid neoplasms: does knowing the origin help to guide treatment? Hematol Am Soc Hematol Educ Program 2016:2432.

5. Klimek VM, Tray NJ. Therapy-related myeloid neoplasms: what's in a name? Curr Opin Hematol 2016;23:161-6.

6. Latagliata R, Petti MC, Fenu S, et al. Therapy-related myelodysplastic syndrome-acute myelogenous leukemia in patients treated for acute promyelocytic leukemia: an emerging problem. Blood 2002;99:822-4.

7. Sasaki K, Jabbour E, Cortes J, et al. Outcome of patients with therapy-related acute myeloid leukemia with or without a history of myelodysplasia. Clin Lymphoma Myeloma Leuk 2016;16:616-24.

8. Arber DA, Orazi A, Hasserjian R, et al. The 2016 revision to the World Health Organization classification of myeloid neoplasms and acute leukemia. Blood 2016;127:2391-405.

9. Hessenauer MRF, Gangat N. Incidence and management of relapsed acute promyelocytic leukemia. In: Blood, editor. 58th ASH Annual Meeting 2016. p. 5179.

10. De Botton S, Chevret S, Sanz M, et al. Additional chromosomal abnormalities in patients with acute promyelocytic leukaemia (APL) do not confer poor prognosis: results of APL 93 trial. Br J Haematol 2000;111:801-6.

11. Wong TN, Ramsingh G, Young AL, et al. Role of TP53 mutations in the origin and evolution of therapy-related acute myeloid leukaemia. Nature 2015;518: 552-5.

12. AE ESS, Chacim S, Ferreira I, et al. Effect of therapy-related acute myeloid leukemia on the outcome of patients with acute myeloid leukemia. Oncol Lett 2016;12:262-8.

13. Zahid MF, Parnes A, Savani BN, et al. Therapy-related myeloid neoplasms what have we learned so far? World J Stem Cells 2016;8:231-42.

14. Zhang L, Wang SA. A focused review of hematopoietic neoplasms occurring in the therapy-related setting. Int J Clin Exp Pathol 2014;7:3512-23.

15. Purizaca J, Meza I, Pelayo R. Early lymphoid development and microenvironmental cues in B-cell acute lymphoblastic leukemia. Arch Med Res 2012;43:89-101. 